\title{
TEMPERATURE CONTROL OF HIGH POWER ELECTRONIC DEVICES AT MINIMUM VENTILATION POWER
}

\author{
Giampietro Fabbri
}

\author{
Department of Energetic, Nuclear, and Environmental Control \\ Engineering, University of Bologna, Viale Risorgimento 2, 40136 \\ Bologna, Italy. E-mail giampietro.fabbri@mail.ing.unibo.it
}

\begin{abstract}
In the present work the cooling of a high power electronic device is studied. The device is in contact with a heat dissipator crossed by air. The air motion through the dissipator is forced by a fan whose supplied power is to be minimized. A finite element dynamic model of the dissipator is firstly created, taking geometrical and physical properties into account as well as steady state experimental data. A simplified model is then obtained, which reproduces the time pattern of the maximum dissipator temperature as a response of the thermal flux removed from the electronic device and of the mass flow rate of the air. Afterwards, the simplified model is utilized to build a control system which allows the electronic device to be correctly cooled at minimum air ventilation power during transition to steady states. Genetic algorithms are used to find the parameters of the finite element model and of the control system. Some functioning conditions of the electronic device are lastly considered and discussed. Copyright ${ }^{\circledR} 2005$ IFAC
\end{abstract}

Keywords: Temperature Control, Heat Transfer, Transient Phenomena, Minimization, Genetic Algorithms

\section{INTRODUCTION}

Many electronic components generate high thermal fluxes which must be dissipated through small surfaces. Due to the evolution of the electronic technology, increasing the commutation frequency and reducing the dimensions of the devices, generated heat fluxes become higher and higher, while component surfaces get smaller and smaller. Therefore, appropriate dissipators are required, in order to remove the heat from the components, keeping their temperature under safety limits (Bar-Cohen and Kraus, 1990; Cesini, et al., 1992).

Many studies have been carried out on the maximization of the dissipator effectiveness, consisting in the capability of removing a heat flux from a surface and dissipating it in a fluid, for a given temperature drop between surface and fluid. The increase in the effectiveness is often accompanied by an increment in the resistance to the fluid motion through the dissipator (Fabbri, 1998; Fabbri, 2000). Therefore, the most efficient heat dissipators require considerable pumping or ventilation power to force the fluid motion.

Since most of electronic devices are usually switched on and off alternatively for time periods of different duration, it is possible to reduce the ventilation power during transition to steady state after the beginning of a working phase. Due to their thermal capacity and inertia, in fact, heat dissipators do not change their temperature instantaneously when the device is switched on, and give just a little amount of the heat flux they 


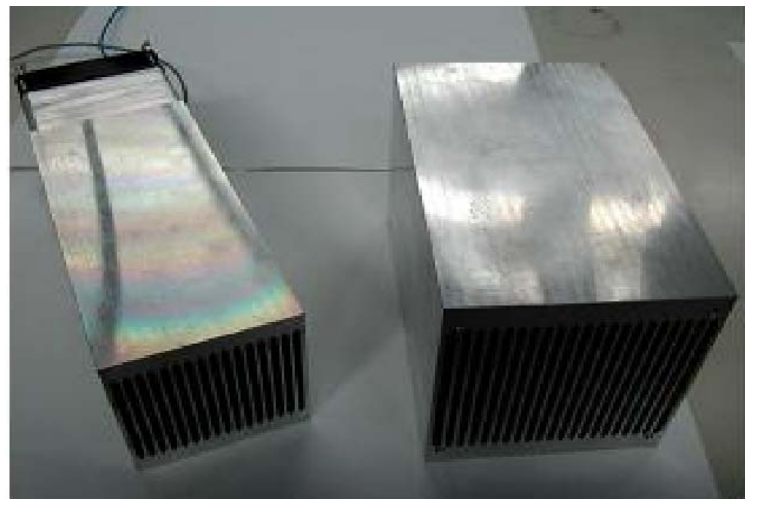

Fig. 1. The aluminium heat dissipator.

receive to the coolant fluid during the initial phase. Therefore, a small rate of fluid is sufficient to remove the initial heat amount from the dissipator.

In this work the thermal behavior of a commercial heat dissipating system for electronic devices is investigated, in order to design a controller which allows the ventilation power to be minimized during switching phases, requiring no direct measure of the cooled device temperature. Such a controller avoids some problems which usually occur in assembling cooler systems when a direct measure of the cooled surface temperature is required. In fact, it is difficult to put a temperature sensor, such as a thermocouple, on the dissipator surface part which is in contact with the electronic component. Inserting the thermocouple simply between the dissipator and the cooled component, would reduce the direct contact between the two bodies and would increase the resistance to the heat transfer. The thermocouple could be located in a cavity or in a hole near to the dissipator surface in contact with the electronic component, but such a manufacturing would be expensive and would even reduce the heat conductance of the dissipator. Moreover, to avoid time expensive wiring in assembling the cooling system, it is useful to insert every parts of the controller in the fan case, avoiding external sensors.

In next sections a finite element dynamic model of the dissipator is firstly created. The values of it parameters are determined by considering steady state experimental data and taking geometrical and physical properties into account. A simplified model for the control system is then obtained. Such a simplified model gives information of the time pattern of the maximum temperature of the dissipator surface where the electronic device is located. On the basis of this information, the control system chooses the appropriate value for the rate of the fluid in order to safely cool the electronic component at minimum ventilation power during transition phases. Different functioning conditions of the electronic device are considered to determine the controller parameters. They are lastly discussed together with the obtained results.
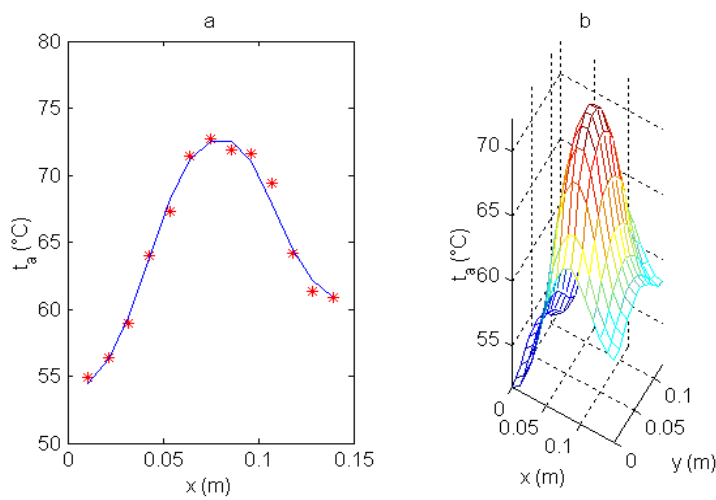

Fig. 2. a) Temperature of the longitudinal mid line of the upper surface of the dissipator: experimental data (stars) and model prediction (continuous line). b) Temperature distribution on the upper surface predicted by the model.

\section{THE COOLING SYSTEM}

The cooling system we investigated is produced by LDS System, Bentivolgio, Bologna, Italy. It is composed of an aluminium dissipator and an electric fan. The dissipator consists of a series of corrugated plates, produced through cold extrusion and joined together using an original pressurebase technique which require no soldering (Figure $1)$. The fan force the air to flow into the channels which are created between the plates. A heat generating component is placed on one of the two dissipator surfaces which are orthogonal to the plates and parallel to the air flow.

We considered a dissipator composed of 22 plates (21 channels), $150 \mathrm{~mm}$ long, $128 \mathrm{~mm}$ high, and $121 \mathrm{~mm}$ wide. Moreover, we studied the case of a component generating a $500 \mathrm{~W}$ heat flux, in contact with the dissipator through a $70 \times 105 \mathrm{~mm}$ rectangular surface, located in the center of the upper dissipator surface and having the long side orthogonal to the air flow. Lastly, we set the fan to the maximum mass flow rate, and the inlet air temperature to $20{ }^{\circ} \mathrm{C}$.

Experimental data concerning these conditions are reported in (Fabbri, et al., 2004). In steady state, the air mass flow rate is equal to $0.024 \mathrm{Kg} / \mathrm{s}$ and the electric power absorbed by the fan is equal to $13 \mathrm{~W}$. Moreover, on the longitudinal mid line of the upper surface of the dissipator the temperature assumes the distribution shown in Figure 2.a

To simulate the time depending behavior of such a cooling system, a finite element model has been created. The corrugated plates have been simulated with flat plates of the same mean thickness $s$. The dissipator has been divided in an upper and a lower part. Moreover, on the upper and the lower dissipator surfaces, a grid of $22 \times 31$ nodes has 

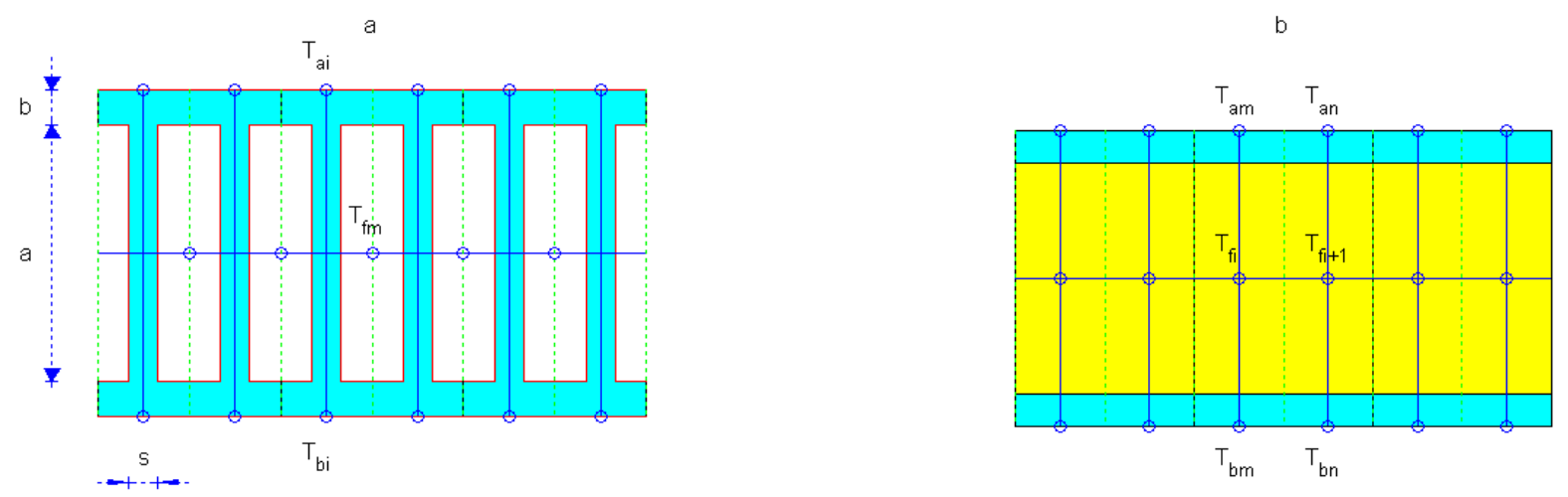

Fig. 3. Node location (o) in the heat dissipator: cross section (a) and lateral section (b).

been drawn (Figures 3.a and 3.b). Every element of the grid has been divided in 4 subelements. Each channel has been divided in 30 portions, by locating 31 nodes in the longitudinal directions.

The model has been created by supposing that the dissipator only exchanges heat with the generating component and with the air flowing inside the channels. Natural convection from the external dissipators surfaces has been neglected.

The heat flux which enter in the dissipator upper part delimited by the 4 subelements surrounding the i-th node can be written as follows:

$$
\begin{aligned}
\dot{Q}_{a_{i}}= & \sum_{k=k_{1}(i)}^{k_{8}(i)} g_{a_{i k}}\left(t_{a_{k}}-t_{a_{i}}\right)+g_{c_{i}}\left(t_{b_{i}}-t_{a_{i}}\right)+ \\
& +\sum_{m=m_{1}(i)}^{m_{2}(i)} g_{h_{i m}}\left(t_{f_{m}}-t_{a_{i}}\right)+\dot{Q}_{i}
\end{aligned}
$$

where $t_{a_{i}}, t_{b_{i}}$, and $t_{f_{m}}$ are the temperature of the dissipator upper part, the lower part, and the fluid, respectively, $g_{a_{i k}}$ are the thermal conductance between node $\mathrm{i}$ and the eight nodes surrounding it on the upper surface, $g_{c_{i}}$ is the conductance between node $\mathrm{i}$ and the nearest node on the opposite dissipator surface, $g_{h_{i m}}$ is the conductance between node $i$ and the nearest nodes in the fluid in the two adjacent channels (Figure 3.a), and $\dot{Q}_{i}$ is the amount of heat flux transferred from the component to the considered dissipator portion. For nodes on the external surface sides or in the corners, index $k$ is limited to consider only 6 or 3 adjacent nodes, respectively, and only one adjacent channel is to be taken into account. $\dot{Q}_{i}$ is proportional to the surface of the dissipator portion which is in contact with the component.

Conductances $g_{a_{i k}}$ and $g_{c_{i}}$ depend on the apparent thermal conductivity $k$ of the composite dissipator, while $g_{h_{m}}$ depend on the convection coefficient $h$ between the channel wall and the fluid (Shah and London, 1974; Shah and Bhatti, 1987). The apparent thermal conductivity of the dissipator results from the heat conductivity of the plates and the contact heat conductance between one plate and another. The convection coefficient increases with the mass flow rate of the fan. Due to the difficulties in calculating $k$ and $h$ analytically or from literature correlations, these parameters have been considered as unknown.

Letting the energy increment per unit of time of the dissipator upper part delimited by the 4 subelements surrounding the $\mathrm{i}$-th node be equal to the heat flux which enters in such a portion, and simplifying eq. (1), the following equation can be written:

$$
\begin{gathered}
C_{t a_{i}} \frac{d t_{a_{i}}}{d \tau}=\sum_{j=j_{1}(i)}^{j_{9}(i)} g_{i j} t_{a_{j}}+g_{c_{i}} t_{b_{i}}+ \\
+\sum_{m=m_{1}(i)}^{m_{2}(i)} g_{h_{i m}} t_{f_{m}}+\dot{Q}_{i}
\end{gathered}
$$

$\tau$ being the time and $C_{t a_{i}}$ the thermal capacity of the considered dissipator portion. Parameters $g_{i j}$ have been derived from $g_{a_{i j}}$ and $g_{h_{i m}}$.

A similar equation can be written for the dissipator portion delimited by the 4 subelements surrounding the $\mathrm{i}$-th node in the lower part:

$C_{t b_{i}} \frac{d t_{b_{i}}}{d \tau}=\sum_{j=j_{1}(i)}^{j_{9}(i)} g_{i j} t_{b_{j}}+g_{c_{i}} t_{a_{i}}+\sum_{m=m_{1}(i)}^{m_{2}(i)} g_{h_{i m}} t_{f_{m}}$

Since the upper and the lower part are symmetrical, the thermal conductance $g_{i j}$ and $g_{h_{i m}}$ are the same on the upper and the lower surface.

Lastly, the heat flux which enter in the channel portion between the $\mathrm{i}$-th and $(\mathrm{i}+1)$-th nodes in the fluid can be written as follows:

$$
\begin{gathered}
\dot{Q}_{f_{i, i+1}}=\sum_{m=m_{1}(i)}^{m_{2}(i)}\left[\frac{g_{h_{m i}}}{2}\left(t_{a_{m}}-t_{f_{i}}\right)+\frac{g_{h_{m i}}}{2}\left(t_{b_{m}}-\right.\right. \\
\left.\left.-t_{f_{i}}\right)\right]+\sum_{n=n_{1}(i+1)}^{n_{2}(i+1)}\left[\frac{g_{h_{n(i+1)}}}{2}\left(t_{a_{n}}-t_{f_{i}+1}\right)+\right. \\
\left.+\frac{g_{h_{n(i+1)}}}{2}\left(t_{b_{n}}-t_{f_{i}+1}\right)\right]+\frac{\dot{M}}{N_{c}} c_{f}\left(t_{f_{i}}-t_{f_{i+1}}\right)
\end{gathered}
$$

where $\dot{M}$ is the total mass flow rate of the fan, $N_{c}$ is the number of channels, and $c_{f}$ is the specific 
heat of the fluid. The last term in Equation (4) represents the energy rate entering in the channel portion with the fluid.

Simplifying eq. (4) and letting the energy increment per unit of time of the channel portion between the $\mathrm{i}$-th and $(\mathrm{i}+1)$-th nodes in the fluid be equal to the heat flux which enters in such a portion, whose mean temperature is assumed to be the mean of $t_{f_{i}}$ and $t_{f_{i+1}}$, the following equation can be written:

$$
\begin{gathered}
C_{t f_{i, i+1}} \frac{d}{d \tau} \frac{t_{f_{i}}-t_{f_{i+1}}}{2}=\sum_{m=m_{1}(i)}^{m_{2}(i)}\left[\frac { g _ { h _ { m i } } } { 2 } \left(t_{a_{m}}+t_{b_{m}}-\right.\right. \\
\left.\left.-2 t_{f_{i}}\right)\right]+\sum_{n=n_{1}(i+1)}^{n_{2}(i+1)}\left[\frac { g _ { h _ { n ( i + 1 ) } } } { 2 } \left(t_{a_{n}}+t_{b_{n}}-\right.\right. \\
\left.\left.-2 t_{f_{i}+1}\right)\right]+\frac{\dot{M}}{N_{c}} c_{f}\left(t_{f_{i}}-t_{f_{i+1}}\right)
\end{gathered}
$$

$C_{t f_{i, i+1}}$ being the thermal capacity of the considered channel portion.

By writing Equation (2), (3), and (5) for every portion of the upper and lower dissipator part and of the channels, the following system is obtained:

$$
\begin{gathered}
C_{t a} * \dot{T}_{a}=A_{a} * T_{a}+B_{a} * T_{b}+C_{a} * T_{f}+D_{a} \dot{Q} \\
C_{t b} * \dot{T}_{a}=A_{b} * T_{a}+B_{b} * T_{b}+C_{b} * T_{f} \\
C_{t f} * I_{m} * \dot{T}_{f}=A_{f} * T_{a}+B_{f} * T_{b}+ \\
+C_{f} * T_{f}+\dot{M} D_{f} I_{d} * T_{f}
\end{gathered}
$$

where vectors $T_{a}, T_{b}$, and $T_{f}$ contain the temperature of the 682 nodes of the dissipator upper part, 682 nodes of the lower part, and 651 nodes in the fluid, respectively, $C_{t a}, C_{t b}$, and $C_{t f}$ are respectively $682 \times 682,682 \times 682$, and $630 \times 630$ diagonal matrices containing thermal capacities, and $I_{m}$ and $I_{d}$ are $630 \times 651$ matrices which give the mean of the difference between the temperature of every two adjacent nodes in the fluid. Lastly, vector $D_{a}$ multiplied by the heat flux generated by the electronic component gives the amount of this flux entered in each node of the dissipator upper part. Under the considered conditions, most of the elements of $D_{a}$ are zero.

In steady state the time derivative of the node temperature are zero, while $\dot{M}$ and $\dot{Q}$ are constant. From system of Equations (6), (7), and (8) a linear system is derived, whose known terms are contained in $T_{f}$, in the 21 positions at the beginning of each channel. After having extracted the elements in these positions from $T_{f}$ and let them be equal to the fan air temperature, the steady state temperature distribution of the cooling system is easily obtained.

In Figure 2.b the steady state temperature distribution obtained on the upper dissipator surface under the considered conditions is shown. The apparent thermal conductivity and the convective heat transfer coefficient in the channels have been found by minimizing the mean square differences between the model predictions and experimental data (Figure 2.a), using a genetic algorithm (Queipo, et al., 1994; Fabbri, 1998; Fabbri, 1999).

In transient phases, system of Equations (6), (7), and (8) gives the time evolution of the cooling system state. Assuming $\dot{M}, \dot{Q}$ and the fan air temperature as the input variables, it is evident that the system is not linear, since some coefficients of the state vector $T_{f}$ depend on the air mass flow rate. Assuming the values of the apparent thermal conductivity and the convective heat transfer coefficient in the channels found in steady state simulation, the differential system of Equations (6), (7), and (8) has then been numerically integrated to obtain the cooling system state evolution as a response of $\dot{M}$ and $\dot{Q}$ at constant fan air temperature.

\section{VENTILATION POWER}

The power absorbed by the fan depends on the air flow rate. Assuming laminar flow inside the channels, the total hydraulic resistance of the dissipator, i. e. the resistance to the air flow, is constant (Parker, et al., 1969). This means that the pressure drop $\Delta p$ in the air before and after crossing the dissipator is proportional to the air volume flow rate $\dot{V}$. Such a pressure drop must be provide by the fun. Therefore, the fluid dynamic power of the fan, i. e. the power transferred from the fan to the fluid, is:

$$
P_{f}=\Delta p \dot{V}=\alpha \dot{V}^{2}
$$

Defining the fan effectiveness $\eta$ as the ratio between $P_{f}$ and the electric power $P_{e}$, this latter results as follows:

$$
P_{e}=\eta \alpha\left(\frac{\dot{M}}{\rho}\right)^{2}=\beta \dot{M}^{2}
$$

$\rho$ being the air density, which can be assumed as a constant, as well as the fan effectiveness. The power absorbed by the fan is then proportional to the square of the air mass flow rate.

Lastly, the mean fan power during the time $\Delta \tau$ is:

$$
\overline{P_{e}}=\frac{1}{\Delta \tau} \int_{0}^{\Delta \tau} \beta \dot{M}^{2} d \tau
$$

\section{THE CONTROL SYSTEM}

A control system has been designed in order to minimize the mean power absorbed by the fan of the cooling system during transition to steady or periodic states. As preannounced in the introduction section, the control system uses a simplified model of the heat dissipator, to obtain information 


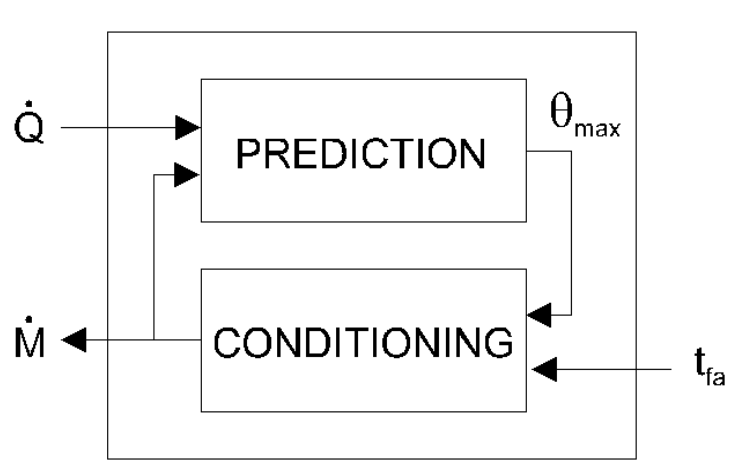

Fig. 4. Block diagram of the control system.

on the maximum temperature $t_{\max }$ of the surface where the heat generating component is located. On the basis of such an information, a conditioning section sets the mass flow rate of the air to the appropriate value (Figure 4).

To create the simplified model, the cooling system response to steps in $\dot{Q}$ in correspondence with different steps in $\dot{M}$ has been investigated. In particular, it has been assumed:

$$
\begin{aligned}
\dot{Q}(\tau) & =U_{Q}(\tau) \dot{Q}_{\max } \\
\dot{M}(\tau) & =U_{M}(\tau) \dot{M}_{\max }
\end{aligned}
$$

where $\dot{Q}_{\max }$ and $\dot{M}_{\max }$ are the values considered in the steady state experiment for $\dot{Q}$ and $\dot{M}$, respectively. Two different steady state values have been assumed for steps in $U_{Q}$ and $U_{M}$ (Figure $5)$ keeping the fan air $t_{f a}$ at $25^{\circ} \mathrm{C}$.

Looking at Figure 5, it is evident that the steady state gain of $\left(t_{\max }-t_{f a}\right)$ with respect to the generated heat flux $\dot{Q}$ and the response time decrease when $\dot{M}$ increases. Therefore, the following state evolution equation has been assigned to the simplified model:

$$
\begin{gathered}
\frac{C_{m}}{1+D_{m} \dot{M}} \frac{d \theta_{\max }}{d \tau}= \\
=-\left(1+B_{m} \dot{M}\right)\left(\theta_{\max }-t_{f a}\right)+A_{m} \dot{M}
\end{gathered}
$$

$\theta_{\max }$ being the model prediction of $t_{\max }$.

Since parameters $C_{m}$ and $D_{m}$ do not influence the steady gain, parameters $A_{m}$ and $B_{m}$ have been firstly determined by minimizing the mean square differences between the simplified model and the cooling system model predictions for steady state. Afterward, parameters $C_{m}$ and $D_{m}$ have been found by minimizing the mean square differences between the time response of the simplified model and the cooling system model (Figure 5). Genetic algorithm have been utilized. The following values have been obtained: $A_{m}=0.1886, B_{m}=33.18$, $C_{m}=295.4, D_{m}=25.04$.

The control problem consists now in finding the time pattern of $U_{M}$ which minimizes the mean ventilation power keeping the maximum temperature of the dissipator surface $t_{\max }$ under a safety
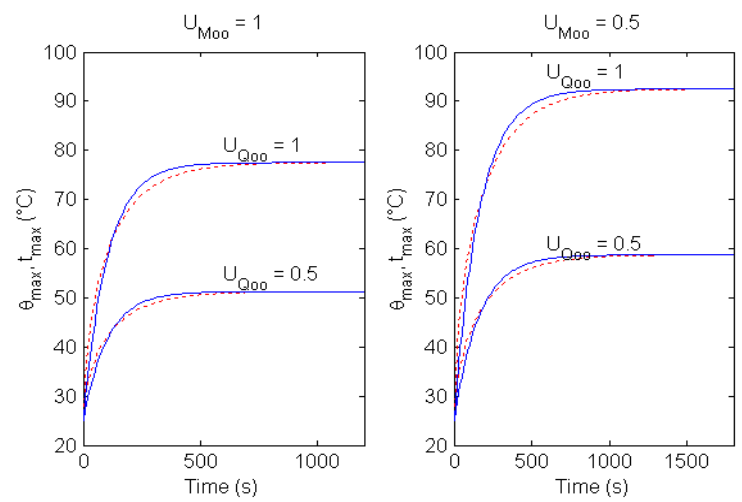

Fig. 5. Simplified model (continuous line) and cooling system model (dashed line) response to step in $\dot{Q}$ and $\dot{M}$.

limit value $t_{\text {saf }}$ which prevents the cooled device from damages. Rewriting eq. (11) taking eq. (13) into account, it is obtained:

$$
\begin{aligned}
\overline{P_{e}} & =\frac{1}{\Delta \tau} \int_{0}^{\Delta \tau} \beta\left(U_{M} \dot{M}_{\max }\right)^{2} d \tau \\
& =P_{e_{\max }} \frac{1}{\Delta \tau} \int_{0}^{\Delta \tau} U_{M}^{2} d \tau
\end{aligned}
$$

where $P_{e_{\max }}$ is the electric power which would be absorbed by the fan if it were always set to the maximum flow rate. The control conditioning section must then be designed in order to minimize the following quantity:

$$
\Pi=\frac{\overline{P_{e}}}{P_{e_{\max }}}=\frac{1}{\Delta \tau} \int_{0}^{\Delta \tau} U_{M}^{2} d \tau
$$

In minimizing $\Pi$ the following constraints must be respected:

$$
\begin{aligned}
& t_{\text {max }} \leq t_{\text {saf }} \\
& U_{M} \in[0,1]
\end{aligned}
$$

Let us remember that the convective conductance $g_{h_{i m}}$ of the cooling system increase with $U_{M}$, and observe that for given values of $g_{h_{i m}}$ in eq. (1) the amount of heat removed from the dissipator is larger when the temperature of this body is higher. This means that the heat can be more efficiently dissipated by keeping the $U_{M}$ as low as possible and $t_{\max }$ as high as possible. Therefore, if $t_{\max }$ were directly measured, an optimum control of such a temperature could be obtained by setting:

$$
U_{M}=\left\{\begin{array}{l}
0, \quad \text { if } t_{\max }<t_{s a f} \\
\frac{A_{m} U_{Q} Q_{\max } /\left(t_{s a f}-t_{f a}\right)-1}{B_{m}}, \\
\text { otherwise }
\end{array}\right.
$$

In this way the fan would be switched off during the transition phase and $t_{\max }$ would be kept equal to $t_{\text {saf }}$ in steady state. The ventilation power would be reduced to the minimum value in both phases.

However, the optimum control is dangerous when the maximum temperature of the dissipator is not directly measured. In fact, if during the life of 

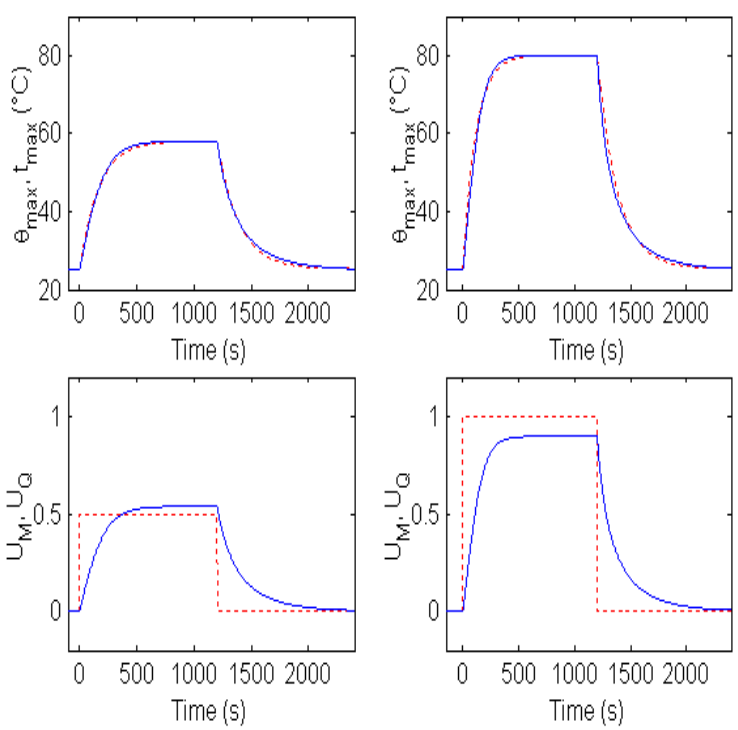

Fig. 6. Time patterns of $t_{\max }(.),. \theta_{\max }(-), U_{Q}$ (..), and $U_{M}(-)$ under condition (a); results obtained by optimizing $P_{c}$
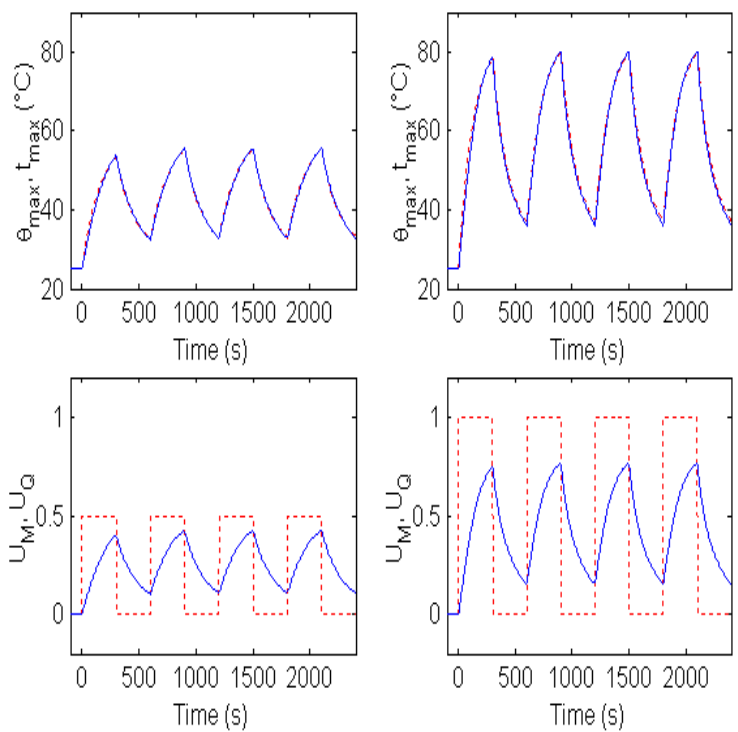

Fig. 7. Time patterns of $t_{\max }(.),. \theta_{\max }(-), U_{Q}$ (..), and $U_{M}(-)$ under condition (b); results obtained by optimizing $P_{c}$.

the cooling system its parameters slightly change, the simplified model can underestimate the real maximum dissipator temperature. In this case, when $t_{\max }$ exceeds the safety limit, the fan is switched off and the amount of heat removed per unit of time is very small. The temperature of the dissipator and the electronic device then increase very quickly to dangerous values.

Therefore, to avoid damaging the electronic component, the control system must be designed in order to always set the fan air flow rate to a relatively high value when the dissipator temperature is expected to be close to the safety limits. In this way, if $t_{\max }$ exceeds the limit it increases more
Table 1.

$\begin{array}{cccccc}P_{c} & A_{m} & B_{m} & C_{m} & D_{m} & \Pi \\ 0.0163 & {[0.1886]} & {[33.18]} & {[295.4]} & {[25.04]} & 0.2508 \\ 0.0164 & {[0.1886]} & {[33.18]} & 339.9 & 48.73 & 0.2470 \\ 0.0262 & 0.0753 & 4.470 & 250.9 & 70.29 & 0.2280\end{array}$

Table 2 .

$\begin{array}{cccccc}P_{c} & A_{m} & B_{m} & C_{m} & D_{m} & \Pi \\ 0.0139 & {[0.1886]} & {[33.18]} & {[295.4]} & {[25.04]} & 0.1573 \\ 0.0129 & {[0.1887]} & {[33.18]} & 310.3 & 90.44 & 0.1475 \\ 0.0247 & 0.0692 & 3.973 & 170.4 & 53.87 & 0.1374\end{array}$

slowly and can settle at the steady state value without becoming too high.

Such a control can be obtained by assigning a proportionality relationship in the conditioning section:

$$
\dot{M}=\dot{M}_{\max } P_{c}\left(\theta_{\max }-t_{f a}\right)
$$

The only parameter $P_{c}$ is to be determined by minimizing the ventilation power during transient phases. However, in order to improve the performance of the control system, we can also consider the simplified model parameters as variables to be determined by minimizing the ventilation power. Therefore, in the next section we investigate how the control system performance change by optimizing only some of its parameters or all.

\section{RESULTS}

The problem of minimizing the ventilation power has been solved by determining the value of the control system parameters with genetic algorithms. Firstly, the only parameter $P_{c}$ has been optimized. The ventilation power minimization has then be improved by also optimizing $A_{m}, B_{m}, C_{m}$, and $D_{m}$, and consequently altering the prediction capability of the simplified model.

In the genetic algorithms the opposite of $\Pi$ has been assumed as an evaluation parameter (Fabbri, 1998). Moreover, the constraints (17) and (18) have been imposed by assigning the following value to $\Pi$ :

$$
\Pi=\left\{\begin{array}{r}
\frac{1}{\Delta \tau} \int_{0}^{\Delta \tau} U_{M}^{2} d \tau, \\
\text { if constraints respected } \\
10\left[1+\left(t_{\max } / t_{\text {saf }}\right)\right]\left(1+\left|U_{M}\right|\right) \\
\text { otherwise }
\end{array}\right.
$$

The quality of the genetic algorithm optimization has been tested by comparison with numerical optimization in case of one parameter to be determined.

Two functioning condition have been considered:

a) the electronic component is switched on for a time longer than the transient duration;

b) the electronic component is switched on and off alternatively for short time periods. 
In both conditions, it has been assumed $t_{f a}=25$ ${ }^{\circ} \mathrm{C}$ and $t_{\text {saf }}=80^{\circ} \mathrm{C}$.

\subsection{Best prediction}

The only parameter $P_{c}$ has been firstly determined by minimizing $\Pi$ during the response to both the $U_{Q}$ time patterns of Figure 6 under condition (a), and of Figure 7 under condition (b). The best values of $P_{c}$, together with the corresponding value of $\Pi$, are reported in Table 1 and 2 for condition (a) and (b), respectively. Figures 6 and 7 also show the corresponding time patterns of $t_{\max }, \theta_{\max }$, and $U_{M}$. As in the following figures, the patterns in the graphics of the first line are determined by the pattern of $U_{Q}$ shown in the graphic below.

It is interesting to observe that the control system dimensioned under condition (a) reduces $\Pi$ to 0.2037, if used under condition (b), while the control system dimensioned under condition (b) does not respect the constraint of Equation (17), if used under condition (a).

In order to test the quality of the genetic algorithm optimization, $\Pi$ has also been numerically minimized by letting $P_{c}$ vary between 0 and $1 /\left(t_{\text {saf }}-\right.$ $\left.t_{f a}\right)$ in $10^{4}$ steps. The difference between the two optimum values found for $P_{c}$ is less than $0.02 \%$.

\subsection{Altered time response}

The performance of the control system improves when parameters $C_{m}$ and $D_{m}$ are assigned together with $P_{c}$ in minimizing $\Pi$. In this way, an error is introduced in the time response of the simplified model.

Figures 8 and 9 shows the time patterns of $t_{\max }$, $\theta_{\max }$, and $U_{M}$ obtained as a response of $U_{Q}$, when parameters $P_{c}, C_{m}$, and $D_{m}$ are optimized by minimizing $\Pi$. Under condition (a) or (b), parameters have been determined considering the response to both the $U_{Q}$ time patterns reported in Figures 8 or 9 , and are reported in Table 1 or Table 2, respectively.

It is evident that the prediction of the simplified model is in delay in Figure 8 and in advance in Figure 9 with respect to the time pattern given by the cooling system model.

Lastly, the control system dimensioned under condition (a) reduces $\Pi$ to 0.1977 , if used under condition (b), while the control system dimensioned under condition (b) still does not respect the constraint of Equation (17), if used under condition (a).

\subsection{Altered time response and steady gain}

The performance of the control system improves even more when all parameters $A_{m}, B_{m}, C_{m}, D_{m}$,
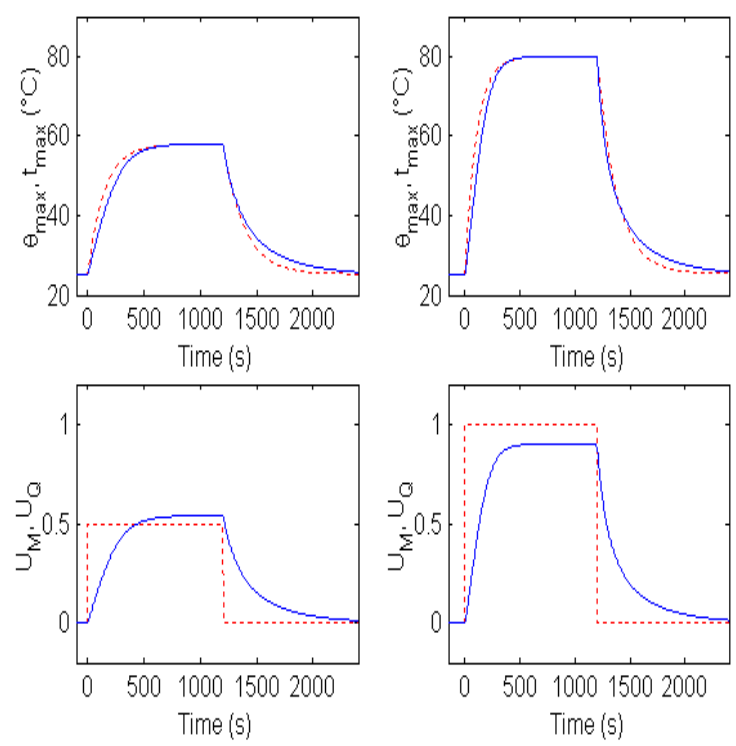

Fig. 8. Time patterns of $t_{\max }(.),. \theta_{\max }(-), U_{Q}$ (..), and $U_{M}(-)$ under condition (a); results obtained by optimizing $P_{c}, C_{m}$, and $D_{m}$.
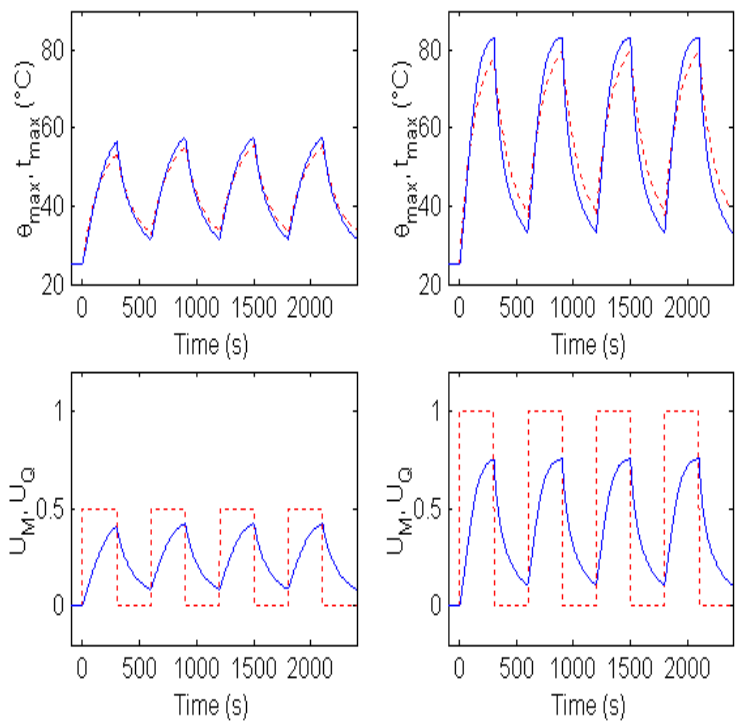

Fig. 9. Time patterns of $t_{\max }(.),. \theta_{\max }(-), U_{Q}$ (..), and $U_{M}(-)$ under condition (b); results obtained by optimizing $P_{c}, C_{m}$, and $D_{m}$.

and $P_{c}$ are assigned by minimizing $\Pi$. In this way, errors are introduced in the time response and in the steady state gain of the simplified model.

Figure 10 and 11 show the time patterns of $t_{\max }$, $\theta_{\max }, U_{Q}$, and $U_{M}$ for condition (a) and (b), respectively. Parameters have been determined considering the response to both the $U_{Q}$ time patterns reported in each figure, and are reported in Table I and Table 2.

It is not easy to distinguish delay or advance in the predictions of the simplified model. Under condition (a), the model underestimates the maximum surface temperature when the step in $U_{Q}$ is lower, and overestimates it when the step 
is higher. On the contrary, under condition (b), the model always underestimates the maximum surface temperature.

It is interesting to observe that the control system dimensioned under condition (a) reduces $\Pi$ to 0.1642 , if used under condition (b), while the control system dimensioned under condition (b) still does not respect the constraint of Equation (17), if used under condition (a).

\section{CONCLUSIONS}

The system proposed to control the temperature of high power electronic devices allows the ventilation power to be noticeably reduced during transition to steady or periodic states. For the case where the electronic device is switched on for a time longer than the transient duration, the control system dimensioned by optimizing only one parameter allows a nearly $75 \%$ reduction in the ventilation power with respect to the case of fan always set to the maximum air flow rate. A further $9.1 \%$ decrement can be obtained by optimizing all parameters of the control system. When the electronic device is switched on and off alternatively for short time periods, a $84 \%$ reduction in the ventilation power is obtainable by optimizing the gain of the control system, and a further $12.6 \%$ decrement by optimizing all parameters.

\section{REFERENCES}

Bar-Cohen, A. and A. D. Kraus (1990). Advances in Thermal modeling of electronic components and systems, 2, 41-107, ASME Press Series, New York.

Cesini, G., R. Ricci, B. Ruggeri (1992). Ottimizzazione di dissipatori di calore alettati per applicazioni elettroniche. Modello numerico e verifica sperimentale. Proc. 10th UIT National Congress, pp. 201-212.

Fabbri, G. (1998). Heat transfer optimization in internally finned tubes under laminar flow conditions, Int. J. Heat and Mass Transfer, 41, n. $10,1243-1253$.

Fabbri, G. (1999). Optimum profiles for asymmetrical longitudinal fins in cylindrical ducts, Int. J. of Heat and Mass Transfer, 42, 511-523.

Fabbri, G. (2000) Heat transfer optimization in corrugated wall channels, Int. J. of Heat and Mass Transfer, 43, 4299-4310.

Fabbri, G., M. Lorenzini, S. Salvigni, (2004). A study on industrial heat sinks for power electronics. Proc. 22nd UIT National Congress, pp. 201-212.

Parker, J., J. Boggs and E. Blick (1969). Introduction to fluid mechanics and heat transfer, Chapt. 4, Addison-Wesley.

Queipo, N., R. Devarakonda and J. A. C. Humphrey (1994) Genetic algorithms for ther-
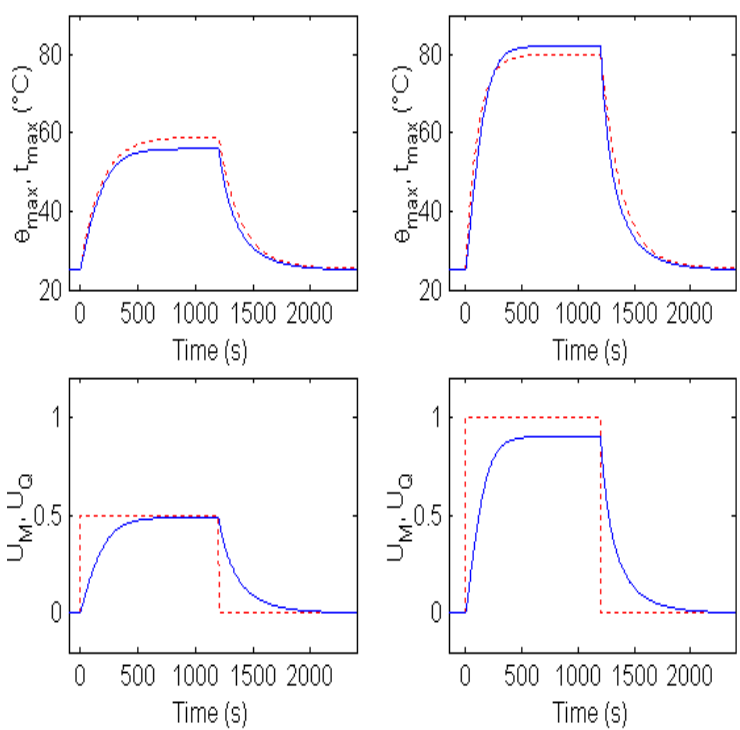

Fig. 10. Time patterns of $t_{\max }(.),. \theta_{\max }(-), U_{Q}$ (..), and $U_{M}(-)$ under condition (a); results obtained by optimizing all control system parameters.
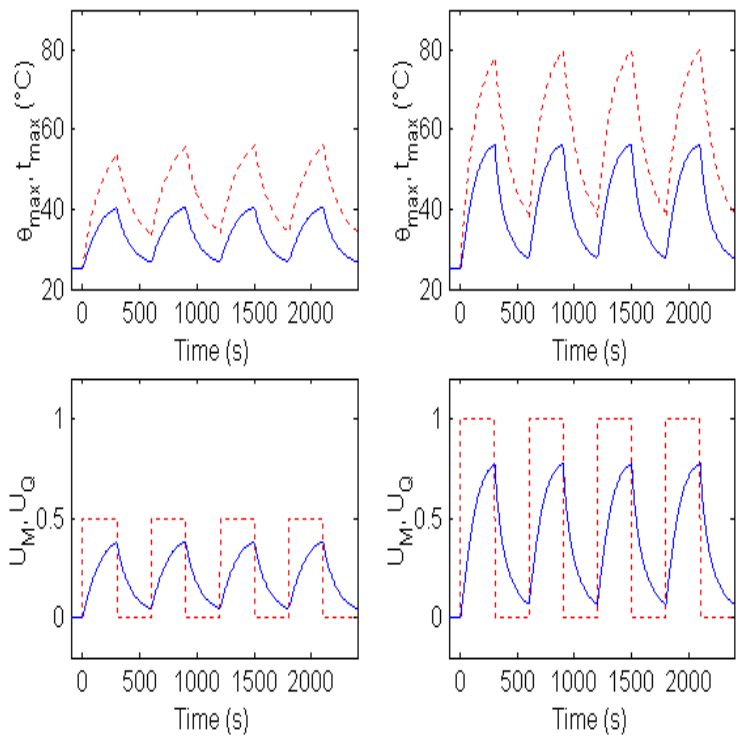

Fig. 11. Time patterns of $t_{\max }(.),. \theta_{\max }(-), U_{Q}$ (..), and $U_{M}(-)$ under condition (b); results obtained by optimizing all control system parameters.

mosciences research: application to the optimized cooling of electronic components, Int. J. Heat and Mass Transfer, 37, n. 6, 893-908.

Shah, R. K. and A. L. London (1974). Thermal Boundary Conditions for Laminar Duct Flow Forced Convection, ASME J. Heat Transfer, 96, 159-165.

Shah, R. K. and M. S. Bhatti (1987), Laminar convective heat transfer in ducts, Handbook of single-phase convective heat transfer, S. Kakac, R. K. Shah, W. Aung Editors, Wiley, New York, 3-12. 